\title{
Prone positioning for acute respiratory failure improved short term oxygenation but not survival
}

\author{
Gattinoni L, Tognoni G, Pesenti A, et al, for the Prone-Supine Study Group. Effect of prone positioning on the survival of \\ patients with acute respiratory failure. N Engl J Med 2001 Aug 23;345:568-73.
QUESTION: Does prone positioning for $\geqslant 6$ hours each day for 10 days improve survival of patients with acute lung injury or the acute respiratory distress syndrome (ARDS)?

\section{Design}

Randomised (allocation concealed), unblinded, controlled trial with 6 months of follow up.

\section{Setting}

28 intensive care units (ICUs) in Italy and 2 in Switzerland.

\section{Patients}

304 patients (mean age 58 y, 68\% men) who were receiving mechanical ventilation and met the following modified criteria for acute lung injury or ARDS: ratio of partial pressure of arterial oxygen $\left(\mathrm{PaO}_{2}\right)$ to fraction of inspired oxygen $\left(\mathrm{FiO}_{2}\right) \leqslant 200$ with a positive end expiratory pressure $(\mathrm{PEEP}) \geqslant 5 \mathrm{~cm}$ of water, or a $\mathrm{PaO}_{2}: \mathrm{FiO}_{2}$ ratio $\leqslant 300$ with a PEEP $\geqslant 10 \mathrm{~cm}$ water; radiographic evidence of bilateral pulmonary infiltrates; and, if measured, pulmonary-capillary wedge pressure $\leqslant 18$ $\mathrm{mm} \mathrm{Hg}$ or absence of clinical evidence of left atrial hypertension. Exclusion criteria were age $<16$ years, cardiogenic pulmonary oedema, cerebral oedema, intracranial hypertension, or clinical conditions contraindicating the use of prone positioning. Follow up at 6 months was $98 \%$.

\section{Intervention}

152 patients were allocated to prone positioning and were continuously kept prone for $\geqslant 6$ hours each day for 10 days. Patients were assessed in the supine position each morning. A change to the prone position was triggered by a $\mathrm{PaO}_{2}: \mathrm{FiO}_{2}$ ratio $\leqslant 200$ with a $\mathrm{PEEP} \geqslant 5 \mathrm{~cm}$ water, or $\mathrm{PaO}_{2}: \mathrm{FiO}_{2}$ ratio $\leqslant 300$ with a PEEP $\geqslant 10 \mathrm{~cm}$ water. 152 patients were allocated to conventional (supine) positioning.

Source of funding: partial funding by Hill-rom Italy.
For correspondence: Dr L Gattinoni, Istituto di Anestesia e

Rianimazione, Ospedale Maggiore

Policlinico-IRCCS,

Milan, Italy.

gattinon@policlinico.mi.it

Abstract and commentary appear in ACP Journal

Club.

\section{Main outcome measures}

Main outcomes were death at 10 days (end of intervention), during ICU stay, and at 6 months. Other outcomes included improvement in respiratory failure and organ dysfunction; presence, site, and severity of pressure sores; and accidental displacement of tracheal or thoracotomy tubes or loss of venous access.

\section{Main results}

Analysis was by intention to treat. The prone and supine groups had similar 10 day mortality, ICU mortality,

Prone $v$ supine positioning for acute respiratory failure*

\begin{tabular}{lllll} 
Mortality & Prone & Supine & RRR (95\% CI) & NNT (CI) \\
\hline At 10 days & $21 \%$ & $25 \%$ & $16 \%(-27$ to 44$)$ & Not significant \\
\hline At ICU discharge & $51 \%$ & $48 \%$ & $5 \%(-16$ to 32$)$ & Not significant \\
\hline At 6 months & $63 \%$ & $59 \%$ & $6 \%(-12$ to 28$)$ & Not significant \\
\hline
\end{tabular}

${ }^{*} \mathrm{CCU}=$ intensive care unit. Abbreviations defined in glossary; RRR, RRI, NNT, NNH, and $\mathrm{Cl}$ calculated from data in article. and 6 month mortality (table), incidence of organ dysfunction, percentage of patients with new or worsening pressure sores, number of days with pressure sores, accidental displacement of tubes, and loss of venous access. Patients in the prone group had slightly greater increases in the $\mathrm{PaO}_{2}: \mathrm{FiO}_{2}$ ratio on morning assessments (average change $63.0 v 44.6, \mathrm{p}=0.02$ ) and more new or worsening pressure sores (2.7 $v 1.9$ per patient, $\mathrm{p}=0.004$ ).

\section{Conclusion}

Prone positioning of patients with acute lung injury or the acute respiratory distress syndrome improved short term oxygenation more than supine positioning, but did not improve survival.

\section{COMMENTARY}

The trial by Gattinoni et al is an important part of an international effort to determine the effect of prone ventilation on survival in patients with acute lung injury. Prone positioning makes good sense physiologically; preclinical and clinical investigations have provided mechanistic information and observational studies, including $>260$ patients with severe acute lung injury, suggest that $60 \%-70 \%$ of patients have an immediate improvement in oxygenation. However, intensive care clinicians are increasingly faced with the frustrating reality that physiological improvement does not always lead to a survival benefit. More importantly, the potential for such serious complications of prone ventilation as inadvertent extubation, loss of venous access and chest tubes, delayed cardiopulmonary resuscitation, and blindness, is clear. As a result, the intensive care world has awaited the completion of this study with great anticipation.

The results, disappointingly, fail to show a survival benefit in patients cared for in the prone position for $\geqslant 6$ consecutive hours each day. However, the true effect of prone ventilation remains unclear. Examination of the confidence intervals reveals that prone positioning for $\geqslant 6$ consecutive hours each day may reduce ICU mortality by as much as $16 \%$ (in which case, we would use it routinely) or may increase ICU mortality by as much as $32 \%$ (in which case, we would not); therefore, more randomised trials are necessary to clarify the role of prone positioning in patients with acute lung injury. In addition, many clinicians believe that 6 hours per day is insufficient to achieve the full potential of prone ventilation. Two randomised trials of prone ventilation are currently underway in Europe, with protocols for longer daily periods of prone ventilation. For now, as the best available evidence suggests no survival benefit and a potential for serious complications, prone positioning should be reserved for patients who are profoundly hypoxemic (as indicated by an intriguing post hoc analysis of these data) or for those enrolled in further clinical research.

Maureen Meade, MD, MSc Assistant Professor Department of Medicine, Division of Critical Care, McMaster University, Hamilton, Ontario, Canada 